\title{
Substructures in the Keplerian disc around the O-type (proto-)star G17.64+0.16 (Corrigendum)
}

L. T. Maud ${ }^{1,2}$, R. Cesaroni ${ }^{3}$, M. S. N. Kumar ${ }^{4}$, V. M. Rivilla ${ }^{3}$, A. Ginsburg ${ }^{5, \star}$, P. D. Klaassen ${ }^{6}$, D. Harsono ${ }^{2}$, Á. Sánchez-Monge ${ }^{7}$, A. Ahmadi ${ }^{8}$, V. Allen ${ }^{9, \star \star}$, M. T. Beltrán ${ }^{3}$, H. Beuther ${ }^{8}$, R. Galván-Madrid ${ }^{10}$, C. Goddi ${ }^{2,11}$, M. G. Hoare ${ }^{12}$, M. R. Hogerheijde ${ }^{2,13}$, K. G. Johnston ${ }^{12}$, R. Kuiper ${ }^{14}$, L. Moscadelli ${ }^{3}$, T. Peters ${ }^{15}$, L. Testi ${ }^{1,3}$, F. F. S. van der Tak ${ }^{16,17}$, and W. J. de Wit ${ }^{18}$

1 European Southern Observatory, Karl-Schwarzschild-Str. 2, 85748 Garching bei München, Germany e-mail: 1maud@eso.org

2 Leiden Observatory, Leiden University, PO Box 9513, 2300 RA Leiden, The Netherlands

3 INAF, Osservatorio Astrofisico di Arcetri, Largo E. Fermi 5, 50125 Firenze, Italy

4 Instituto de Astrofísica e Ciências do Espaço, Universidade do Porto, CAUP, Rua das Estrelas, 4150-762 Porto, Portugal

5 National Radio Astronomy Observatory, 1003 Lopezville Road, Socorro, NM 87801, USA

${ }^{6}$ UK Astronomy Technology Centre, Royal Observatory Edinburgh, Blackford Hill, Edinburgh EH9 3HJ, UK

7 I. Physikalisches Institut der Universität zu Köln, Zülpicher Str. 77, 50937 Köln, Germany

8 Max Planck Institute for Astronomy, Königstuhl 17, 69117 Heidelberg, Germany

9 NASA Goddard Space Flight Center, Greenbelt, MD 20771, USA

10 Universidad Nacional Autónoma de México, Instituto de Radioastronomía y Astrofísica, Morelia, Michoacán 58089, Mexico

11 Department of Astrophysics/IMAPP, Radboud University, PO Box 9010, 6500 GL Nijmegen, The Netherlands

2 School of Physics and Astronomy, University of Leeds, Leeds LS2 9JT, UK

13 Anton Pannekoek Institute for Astronomy, University of Amsterdam, Science Park 904, 1098 XH Amsterdam, The Netherlands

14 Institute of Astronomy and Astrophysics, University of Tübingen, Auf der Morgenstelle 10, 72076 Tübingen, Germany

15 Max-Planck-Institut für Astrophysik, Karl-Schwarzschild-Str.1, 85748 Garching, Germany

${ }_{16}$ Kapteyn Astronomical Institute, University of Groningen, The Netherlands

17 SRON, Landleven 12, 9747 AD Groningen, The Netherlands

18 European Southern Observatory, Alónso de Cordova 3107, Vitacura, Casilla, 19001 Santiago de Chile, Chile

A\&A, 627, L6 (2019), https://doi .org/10.1051/0004-6361/201935633

Key words. stars: formation - stars: protostars - stars: massive - stars: winds, outflows - stars: pre-main sequence - errata, addenda

We noticed that the $x$-axes for Fig. 2d and Fig. A.1a,b were incorrectly labelled "Right Ascension Offset (mas)" when referring to the position-velocity (PV) diagrams. The correct label is "Position Offset (mas)".

$\star$ Jansky fellow.

$\star \star$ NASA postdoctoral program fellow. 